\title{
CIUDADES INTELIGENTES Y APPS PARA LA CIUDADANÍA: ANÁLISIS DE CASOS PIONEROS EN ESPAÑA
}

\section{Smart Cities and Apps for Citizenship: Analysis of Innovation Cases in Spain \\ Cidades Inteligentes e Apps para a Cidadania: Análise de Casos Pioneiros em Espanha}

Teresa Barceló-Ugarte, Universidad San Pablo CEU de Madrid (España) tbarcelo@ceu.es

Francisco Cabezuelo-Lorenzo, Universidad Complutense de Madrid (España)

fcabezuelo@ucm.es

María Sánchez-Martínez, Universidad San Pablo CEU de Madrid (España)

msanmar.fhum@ceu.es

\section{Recibido: 24 de agosto de 2016}

\section{RESUMEN}

Las ciudades inteligentes (smart cities) representan la evolución natural de la urbe tradicional gracias a la aplicación de las nuevas tecnologías de la información y de la comunicación a sus procesos de funcionamiento cotidiano. En la actualidad, se están implantando infraestructuras y dispositivos dirigidos a un amplio marco de acción de muy variada influencia en donde eficiencia energética, accesibilidad, movilidad, reducción de la contaminación o recursos sostenibles son los ítems más repetidos. En este proceso de transformación integral de la ciudad, los 


\section{DISERTACIONES}

AVANCES

Anuario electrónico de estudios en Comunicación Social

ISSN: $1856-9536$

Doi: http://dx.doi.org/10.12804/revistas.urosario.edu.co/disertaciones/a.5106

Volumen 10, Número 2 / Julio-diciembre 2017

Versión PDF para imprimir desde

http://revistas.urosario.edu.co/index.php/disertaciones

ciudadanos y los contenidos que van dirigidos a ellos deberían ser prioritarios, sin embargo parecen estar ausentes en la gran mayoría de estudios e investigaciones al respecto. Este trabajo centra su atención en el análisis de las aplicaciones digitales de las ciudades españolas de Valencia, A Coruña y Burgos, pioneras en el área de las smart cities y sus contenidos, así como en las innovaciones que mejoran su integración en el nuevo ecosistema digital.

Palabras clave: ciudades inteligentes, España, teléfonos inteligentes, tecnologías de la información y de la comunicación.

\section{ABSTRACT}

Smart cities represent the natural development of the traditional major city because of new Information and Communications Technology applied to their daily operating processes. In the cities' process of comprehensive transformation, the citizens and the content aimed at them should appear in order of priority. However, they seem to be missing in the large majority of studies and research on the topic. This article focuses its attention on the analysis of digital apps by pioneering Spanish cities such as Valencia, Coruña and Burgos, in the field smart city apps and their content, as well as innovations that improve their integration into the new digital ecosystem.

Keywords: Smart Cities, Spain, apps, smartphones, information and communication technology.

\section{RESUMO}

As cidades inteligentes (smart cities) representam a evolução natural da cidade graças tradicionais para a aplicação das Novas Tecnologias de Informação e Comunicação aos seus processos de operação diários. Atualmente, eles estão sendo implantadas infraestruturas e dispositivos destinados a um amplo quadro de acção influência variada, onde a eficiência energética, acessibilidade, mobilidade, redução da poluição e dos recursos sustentáveis são os itens mais utilizadas. Neste processo de transformação abrangente da cidade, os cidadãos e os conteúdos são direcionados a eles devem aparecer em ordem de prioridade, mas, no entanto, parecem estar ausentes na grande maioria dos estudos e investigações. Este trabalho se concentra na análise de aplicações digitais pioneiros cidades espanholas (Valencia, A Corunha, Burgos) na área de Cidades Inteligentes e seu conteúdo, bem como as inovações que melhoram a sua integração no novo ecossistema digital.

Palavras-chave: cidades inteligentes, Espanha, smartphones, tecnologia da informação e comunicação. 


\section{Introducción y justificación}

Los avances tecnológicos que se han producido en el último cuarto de siglo y principalmente el desarrollo de internet han propiciado el acceso de los ciudadanos a una cantidad infinita y variada de contenidos digitales desarrollados no solo a través de iniciativas privadas sino también públicas. Redes sociales, plataformas digitales, dispositivos móviles, tabletas, la mensajería instantánea o los periódicos digitales han creado un nuevo ecosistema de relaciones informativas, políticas y ciudadanas, según Manuel Castells (2008, pp. 13-14). Una de las consecuencias de la digitalización, ya sea texto, audio, video o imágenes, bien a través de la transformación de contenido analógico en un conjunto de dígitos binarios o directamente por la captura digital de la información, ha motivado que los contenidos puedan ser vistos y consumidos a través de diferentes dispositivos digitales propiciando una convergencia tecnológica y multiplataforma (Sánchez-Martínez e Ibar-Alonso, 2015, p. 87).

En el área de las nuevas tecnologías de la información y de la comunicación, han surgido tres nuevas realidades como son el Internet de las Cosas (Internet of Things, IOT), la comunicación Machine to Machine (M2M) y el mundo Big Data, entendidos como "volúmenes masivos y complejos de información, tanto estructurada como no estructurada, que es recogida durante cierto período de tiempo y que requiere de métodos computacionales para extraer conocimiento" (Arcila-Calderón et al., 2016, p. 624). Estos tres macroconceptos "capitalizan hoy el planteamiento y desarrollo de la innovación" en este sector (Bonete, 2015, p. 1).

En este contexto, se sitúa el fenómeno de las ciudades inteligentes ${ }^{1}$ (en español) o smart cities (en inglés), consideradas ya por muchos una realidad llamada a convertirse en el único hábitat conocido ya por esa generación que Marc Prensky (2001) denominó la de los "nativos digitales". Como una fase más de ese proceso comunicativo entre máquinas, aplicable a nuestro día a día, y que genera durante y tras la ejecución del protocolo electrónico en cuestión cantidades ingentes de información analizable y provechosa para la mejora de calidad de vida de las personas se sitúan también, y en lugar privilegiado, las llamadas smart cities (Bonete, 2015, pp. 1-7). Además, el mundo de las ciudades inteligentes tiene una profunda relación con el mundo del tratamiento de datos ligado a las Ciencias de la Información y de la Comunicación, ya que "la mayoría de las iniciativas tecnológicas en materia de smart cities se fundamentan en el desarrollo de aplicaciones que analizan y generan nuevos datos útiles para la ciudadanía" (Cabezuelo-Lorenzo et al., 2016, p. 104), fruto del análisis de los Big Data ahora tan en boga.

En este entorno, la aparición de dispositivos portátiles como las tabletas y los teléfonos inteligentes (smartphones) han hecho florecer un universo de aplicaciones que ayudan a los usuarios en su vida cotidiana a acceder a una amplia tipología de contenidos como son el ocio, la cultura, el turismo, la salud, la educación o el comercio electrónico. Evidentemente no todas las aplicaciones que se desarrollan son útiles o interesantes y en ocasiones no son más que una prolongación adaptada de los contenidos de la página web corporativa. Todos estos desarrollos son fundamentales a la hora de alcanzar la gestión inteligente de las ciudades y deben estar en un lugar preferencial en los calendarios acciones de empresas y administraciones públicas en su deseo de acercarse a la ciudadanía.

1 Conforme a la tradición científica en este sector, este trabajo utiliza indistintamente el término inglés "smart cities" como su equivalente en lengua española "ciudades inteligentes" tanto en su forma plural como en el singular con el fin de dar a conocer tanto la terminología anglosajona como la hispana y para evitar la repetición innecesaria de una única forma.

\section{7}


Desde el momento en el que la ciudadanía empieza a tener acceso a internet, comienzan la navegación, las descargas y las visualizaciones de contenidos que van conformando un tejido digital que permite conocer las necesidades, usos y gustos de los usuarios. Así, dentro de los cambios y avances que se están produciendo, las ciudades se han convertido en un sistema en tiempo real que genera grandes cantidades de datos; su uso inteligente, de la mano de las nuevas tecnologías, pueden facilitar el aprovechamiento de todo el potencial que las ciudades inteligentes encierran, no solo en lo que a eficiencia y sostenibilidad ambiental se refiere sino también en la estimulación de la innovación para la vida cotidiana en todos sus aspectos y con la creación de aplicaciones que permitan su difusión, accesibilidad, modelos de negocio nuevos (Cabezuelo-Lorenzo et al., 2016, p. 108) y que, por supuesto, permitan avanzar tanto a las ciudades como sus habitantes.

\section{Smart cities \& smart citizens}

Las infraestructuras basadas en la tecnología contribuyen a que las ciudades sean más eficaces en cuanto a la optimización de las necesidades de los ciudadanos, además de contribuir a su desarrollo socioeconómico. La nueva economía, por tanto, se gana un lugar en las ciudades a través de las ciudades inteligentes con soluciones innovadoras que a su vez consiguen una mayor calidad de vida para sus ciudadanos.

Para la ciudadanía, disponer de una smart city ayuda a la gestión automática y eficiente de las infraestructuras y servicios urbanos, lo que redunda en la reducción del gasto público, la mejora de la calidad de los servicios prestados, la mejora de la información a los ciudadanos y la mejora en la toma de decisiones. Además, la plataforma Smart City constituye en sí misma, una vía para la innovación al favorecer la incubación de nuevos negocios e ideas (Fundación Telefónica, 2011).

En el contexto de competitividad y de innovación de las smart cities es necesaria la existencia de avances específicamente dirigidos a los ciudadanos a la hora de fomentar su integración en el ecosistema digital de las ciudades inteligentes con la finalidad de permitirles el aprovechamiento de las Nuevas Tecnologías de la Información y de la Comunicación (NTIC) dentro de las ciudades inteligentes. Además, es necesario encontrar un valor añadido a través de la participación y la innovación, como parece ser objetivo clave del Programa Marco de Investigación e Innovación de la Unión Europea Horizonte 2020 (MECD, 2014) y esto es posible en el contexto de cambio propiciado por las smart cities y sus tres motores (M2M, IOT y Big Data) que brindan una oportunidad única e inmejorable para fomentar el acceso, la difusión y promoción del turismo, el patrimonio cultural, la educación, el comercio o la salud a través de medios digitales electrónicos.

Existen tres perspectivas que conforman el panorama de la internet del futuro y el desarrollo de las ciudades (Schaffer, Komninos y Pallot, 2011) que resumen los retos infraestructurales y el papel que toman los agentes implicados en el proceso de transformación hacia las smart cities a partir de cuatro variables: los agentes, las prioridades, los recursos y las políticas.

La primera perspectiva es la de internet del futuro, donde los agentes implicados deben ser los investigadores y las compañías TIC; las prioridades, los retos técnicos; los recursos, las instalaciones para la experimentación, un entorno piloto y las propias tecnologías; y por último, las políticas deben apoyar la creación de instalaciones avanzadas, bancos de prueba y la investigación experimental.

\section{8}


La segunda perspectiva es la del desarrollo de las ciudades y el urbanismo, donde los agentes deben ser los políticos, las plataformas de ciudadanos y las asociaciones de empresarios. Las prioridades deben centrarse en el desarrollo urbano, las infraestructuras esenciales y la creación de nuevos negocios. Los recursos deben basarse en el desarrollo de marcos de política urbana, bienes organizacionales y planes de desarrollo y, por último, las políticas municipales estimularán la innovación, la creación de nuevas empresas y la contratación innovadora.

La tercera perspectiva es la de los ecosistemas de innovación impulsados por los usuarios, donde los agentes implicados deben ser por un lado directores de laboratorios en vivo y por otro, ciudadanos, gobiernos, empresas e investigadores como cocreadores; las prioridades, la innovación abierta -fundamentalmente impulsada por los usuarios-y el fomento de la participación de los ciudadanos. Se debe poder contar con los recursos necesarios, como instalaciones de laboratorios en vivo donde haya metodología, herramientas, e infraestructura física. Por último, las políticas deben ser aquellas que impulsen la innovación abierta y colaborativa.

Sin embargo, parece que no es tan fácil generar estrategias y modelos digitales de actuación en este sentido y dentro del contexto de las smart cities. En primer lugar, es difícil por todas las áreas que abarca y, en segundo lugar, por la gestión de intangibles que representa en algunos casos como en el patrimonio, la educación o la cultura digital, especialmente cuando todas las ciudades están generando estrategias de promoción y posicionamiento cultural como destinos turísticos (Herranz de la Casa, 2015, pp. 217-229). Pero es necesario que, a través de estos nuevos impulsos y mejoras que sirven para generar innovación y riqueza con base en lo que las nuevas tecnologías permiten, se pueda contribuir a la construcción de ciudades en donde su identidad, patrimonio y los bienes y servicios que la componen puedan ser transmitidos, gestionados y disfrutados tanto por los ciudadanos que la integran como por los que la visitan.

La clave para poder garantizar el éxito de una ciudad inteligente son los ciudadanos que en ella habitan, por lo que hay que prestar atención especial a grupos específicos como son los menores y especialmente la tercera edad (González-Oñate et al., 2015, pp. 19-28). "Los ciudadanos de más edad son los que, por lo general, presentan más problemas a la hora de manejar las nuevas tecnologías. De este modo, y dado que el envejecimiento es una constante en la sociedad actual, se plantea un problema en lo que al funcionamiento de la smart city se refiere: una ciudad inteligente será tan eficiente como amplio sea el espectro de población capaz de interpretar y utilizar sus instrumentos tecnológicos" (Enerlis, Ernst y Young, 2012, p. 71).

En este sentido son necesarias políticas de fomento y acción que permitan, tanto desde los poderes públicos como de iniciativas privadas, el acceso y comprensión de todo lo que rodea a las ciudades inteligentes. De nada sirve que se realicen nuevos desarrollos para unos ciudadanos que difícilmente entienden y manejan los lenguajes y aplicaciones digitales. Por fortuna, en la actualidad, aunque no siempre con éxito, conviven políticas de apoyo a estos grupos específicos y otras que van desde sistemas de mejora del ahorro energético hasta incluso el aprovechamiento de los datos que los propios ciudadanos se brindan como sensores en lo que a transporte público se refiere.

Del mismo modo, las administraciones fomentan la participación ciudadana a través de las aplicaciones que han desarrollado para la gestión telemática y, por último, la creación de espacios online y redes sociales que permiten una comunicación fluida así como la retroalimentación. Tal y como queda establecido en el Convenio de Aarhus (BOE, 2005), en la sociedad, los ciudadanos deben gozar del derecho de tomar parte en los procesos de participación pública en ámbitos como el desarrollo de planes y programas, evaluación de proyectos o en el desarrollo de la legislación así como a que la información resulte accesible. Por tanto, en el contexto de las ciudades 
inteligentes donde existen dificultades añadidas por su propia idiosincrasia tecnológica, no todos los ciudadanos están en disposición de poder acceder a estos contenidos, bien sea por falta de alfabetización digital ya sea por edad o nivel sociocultural o por carencias derivadas de su falta de poder adquisitivo.

\section{Hipótesis, objetivos y metodología}

En el caso de España, desde el año 2011, a partir de la firma del Manifiesto por las Ciudades Inteligentes. Innovación para el progreso, el compromiso era crear una red abierta para dar pie al progreso económico, social y empresarial de las ciudades a través de la innovación y el conocimiento a través de las TIC. Por este motivo se crea la Red Española de Ciudades Inteligentes (RECl) que tiene la responsabilidad de desarrollar un modelo de gestión sostenible y mejorar la calidad de vida de los ciudadanos. Desde ese momento, se han ido adhiriendo ciudades y ayuntamientos hasta un total de 65 en toda España (RECl, 2015).

En 2014 más de 21,4 millones de españoles accedieron a internet a través de dispositivos móviles (Fundación Telefónica, 2015) por tanto, las empresas y las administraciones públicas deberían ser los agentes facilitadores e integradores de la sociedad y de sus ciudadanos hacia los contenidos digitales. La hipótesis de partida es conocer la oferta real que existe, a partir de la creación de RECl, de aplicaciones que fomenten la participación ciudadana, que promuevan la accesibilidad a los contenidos de la ciudad y, por supuesto, que difundan todos el avances y mejoras que se realizan, per se y para con sus ciudadanos, en las ciudades tomadas como muestra.

Por tanto, los objetivos principales son conocer qué han hecho las ciudades de RECI por brindar una oferta de apps ciudadanas y comprobar la operatividad, funcionalidad y servicio que presta al ciudadano.

La investigación se realizó durante los meses de octubre, noviembre y diciembre de 2015, meses en los que a través de la observación se guardaron las unidades de análisis. Una vez establecidos los objetivos de este estudio, se seleccionaron los contenidos a analizar teniendo en cuenta su interés para los ciudadanos y que estuvieran dentro de la línea de investigación que sigue el proyecto en el que se enmarca este artículo. Estos fueron (a) Cultura y deporte, (b) Salud y teleasistencia, (c) Turismo y ocio, Educación, (d) Sistemas inteligentes de transportes y e-commerce y plataformas de pago NFC. Se acudió a la RECI para seleccionar cuáles serían las ciudades españolas sobre las que se analizarían las aplicaciones. Las ciudades pioneras en España ${ }^{2}$ seleccionadas han sido La Coruña/A Coruña (Galicia), Burgos (Castilla y León), Valencia/València (Comunidad Valenciana/Comunitat Valenciana) y Valladolid (Castilla y León). La justificación es que la RECI se organiza alrededor de grupos de trabajo y, en este caso, los grupos a seguir para nuestro análisis eran:

- Grupo 1, centrado en temáticas sobre innovación social entre las que se encuentran cultura y deporte, salud y teleasistencia, turismo y ocio, y educación. La ciudad que lidera este grupo de trabajo es La Coruña, por eso fue la primera elegida para revisar los contenidos que habían desarrollado con tal fin.

2 Junto a la lengua española o castellano son lenguas cooficiales el gallego en Galicia o el valenciano en la Comunidad Valenciana, motivo por el que este trabajo recoge el nombre de La Coruña/A Coruña y Valencia/València tanto en castellano como en sus respectivas lenguas autonómicas. 
- Grupo 4, desarrolla temáticas sobre movilidad urbana. En este caso nos interesaba la línea de trabajo referida a los sistemas inteligentes de transportes, de esta manera, las ciudades que lideraban este grupo de trabajo, Burgos y Valladolid, fueron incorporadas al análisis.

- Grupo 5, centrado en temáticas sobre Gobierno, Economía y Negocios. De este grupo nos interesaba la línea de trabajo centrada en e-comercio y plataformas de pago NFC. En este caso, la ciudad que lidera el grupo es Valencia.

Así, finalmente, La Coruña, Burgos, Valladolid y Valencia son las ciudades de España en las que se centra la metodología.

Seleccionadas las ciudades y las líneas de interés ciudadano, se realizó un análisis de contenido en el que se examinaron los sitios web de dichos ayuntamientos, publicaciones relacionadas y su presencia en las tiendas de aplicaciones de Android (Google Play) y de Apple (App Store) y sus desarrolladores. A partir de la información obtenida se pudo acotar la posible dispersión de aplicaciones existentes y seleccionar las cuatro aplicaciones que se han analizado: Muévete es Burgos, Eventos Smart Coruña, Visitas Guiadas Coruña y App Valencia.

Aunque algunos de los ayuntamientos que formaban parte del estudio habían desarrollado otras aplicaciones, se seleccionaron exclusivamente aquellas sobre las que se pudiera analizar el contenido expuesto anteriormente y que estuviera basado en las líneas de investigación del proyecto; se desecharon las que no correspondían con las líneas especificadas en los grupos de trabajo, motivo por el que, aunque Valladolid es una ciudad inteligente y pionera en España, no se ha tomado su aplicación como válida para este análisis comparativo en el análisis de casos, pero sí para otros aspectos.

Por último se definieron las variables cualitativas del análisis (V) para cada una de estas aplicaciones. Dichas variables permitieron analizar de forma homogénea la información de las aplicaciones:

V1) Ciudad de procedencia: para localizar el destino de cada una de las aplicaciones, aunque en este caso todas ellas incluyen el nombre de la ciudad de procedencia en su título.

V2) Tipo de contenido: para conocer si la temática es sobre Cultura y deporte, Salud y teleasistencia, Turismo y ocio, Educación, Sistemas inteligentes de transportes o e-commerce y plataformas de pago NFC.

V3) Desarrollador: ofrece información sobre el organismo que ha desarrollado la aplicación.

V4) Tipo de servicio: explicación sobre el contenido de la aplicación.

V5) Versión de la aplicación: donde se puede comprobar el interés del desarrollador por mantener actualizada la aplicación e incluir mejoras.

V6) Número de descargas: permite conocer el interés del público hacia ese contenido y la difusión que ha realizado el desarrollador para dar a conocer el producto (información disponible únicamente en Google Play).

V7) Público al que se dirige: información sobre la recomendación de edad para el acceso a los contenidos que se publican en la aplicación.

V8) Evaluación de usuarios: aunque no todos los usuarios que descargan aplicaciones opinan sobre ellas, los comentarios que vierten en la red ayudan a otras personas que pueden estar interesadas en la temática de la aplicación.

v9) Otras aplicaciones del desarrollador: para conocer la actividad de los ayuntamientos analizados en el entorno digital.

V10) Además de estas variables, en cada una de las aplicaciones analizadas se estudió su diseño para conocer su nivel de operatividad y accesibilidad. 
Los contenidos de las aplicaciones analizadas se descargaron en el otoño de 2015, tanto para el sistema operativo Android como para el sistema operativo iOS, y fueron analizadas durante el mes de enero de 2016.

Este trabajo parte de un principio que estima que si las nuevas tecnologías y los dispositivos digitales tienen la estructura para soportar la innovación necesaria a la hora de llegar a los ciudadanos, es preciso conocer el grado en que las empresas y sobre todo las administraciones públicas municipales aprovechan en este ámbito. En este sentido, hace hincapié en el estudio de las aplicaciones que fomenten no solo la participación ciudadana, sino también en el de aquellas que promueven la accesibilidad, la integración del conocimiento y, por supuesto, la difusión de todos los avances y mejoras que se realizan en las ciudades per se y para con sus ciudadanos. Por ese motivo, se toman las aplicaciones móviles (apps) como objeto de estudio, entendidas como el máximo exponente actual, junto con las redes sociales, de la nueva sociedad digital.

\section{Análisis y resultados}

El análisis se ha realizado sobre las cuatro aplicaciones que cumplían los requisitos desarrollados en la metodología: Muévete es Burgos, Eventos Smart Coruña, Visitas Guiadas Coruña y App Valencia.

\section{Tabla 1}

\begin{tabular}{|c|c|c|c|c|}
\hline & $\begin{array}{l}\text { Muévete es } \\
\text { Burgos }\end{array}$ & $\begin{array}{c}\text { Eventos Smart } \\
\text { Coruña }\end{array}$ & $\begin{array}{c}\text { Visitas Guiadas } \\
\text { Coruña }\end{array}$ & App Valencia \\
\hline $\begin{array}{l}\text { Ciudad de } \\
\text { procedencia }\end{array}$ & Burgos & A Coruña & A Coruña & Valencia \\
\hline Tipo de contenido & Transporte & Cultura y ocio & Turismo & $\begin{array}{l}\text { Comercio } \\
\text { electrónico }\end{array}$ \\
\hline Desarrollador & $\begin{array}{c}\text { Ayuntamiento de } \\
\text { Burgos }\end{array}$ & Concello da Coruña & Concello da Coruña & $\begin{array}{c}\text { Ajuntament de } \\
\text { València }\end{array}$ \\
\hline $\begin{array}{l}\text { Versión de la } \\
\text { aplicación }\end{array}$ & $\begin{array}{l}\text { 1.1.0 (Android) } \\
1.2 .0 \text { (iOS) }\end{array}$ & $\begin{array}{l}\text { 1.0.2 (Android) } \\
1.0 \text { (iOS) }\end{array}$ & 1.1.2 (Android) & $\begin{array}{c}1.0 .44 \text { (Android) } \\
1.2 .6 \text { (iOS) }\end{array}$ \\
\hline $\begin{array}{l}\text { Número de } \\
\text { descargas }\end{array}$ & Más de 100 & Más de 5.000 & Más de 1.000 & Más de 10.000 \\
\hline $\begin{array}{l}\text { Público al que se } \\
\text { dirige }\end{array}$ & $\begin{array}{l}\text { PEGi } 3 \text { (Android) } \\
\text { Más de } 4 \text { (iOS) }\end{array}$ & $\begin{array}{l}\text { Sin clasificar } \\
\text { (Android) } \\
\text { Más de } 4 \text { (iOS) }\end{array}$ & $\begin{array}{l}\text { Sin clasificar } \\
\text { (Android) }\end{array}$ & $\begin{array}{l}\text { PEGI } 3 \text { (Android) } \\
\text { Más de } 4 \text { (iOS) }\end{array}$ \\
\hline $\begin{array}{l}\text { Evaluación de } \\
\text { usuarios }\end{array}$ & $\begin{array}{c}3,4 \text { sobre } 5 \\
\text { (basado en } \\
10 \text { usuarios) } \\
\text { (Android) }\end{array}$ & $\begin{array}{c}\text { 4,1 sobre } 5 \text { (basado } \\
\text { en } 101 \text { usuarios) } \\
\text { (Android) } \\
5 \text { sobre } 5 \text { (basado } \\
\text { en } 10 \text { usuarios) } \\
\text { (iOS) }\end{array}$ & $\begin{array}{c}\text { 4,1 sobre } 5 \text { (basado } \\
\text { en } 32 \text { usuarios) } \\
\text { (Android) }\end{array}$ & $\begin{array}{c}\text { 3,9 sobre } 5 \text { (basado } \\
\text { en } 209 \text { usuarios) } \\
\text { (Android) }\end{array}$ \\
\hline
\end{tabular}

Fuente: Elaboración propia. 
Para presentar los resultados obtenidos, estos se analizarán por ciudades. Burgos y Valladolid lideran el Grupo 4 de trabajo de la $\mathrm{RECl}^{3}$ que desarrolla proyectos sobre movilidad urbana; en concreto sobre movilidad eléctrica y sistemas inteligentes de transporte. En este sentido, el Ayuntamiento de Valladolid en su página web (www. valladolid.es) apenas ofrece información referente a smart cities y está desactualizada. A pesar de encabezar junto a Burgos el grupo de trabajo 4, no se ha encontrado ninguna aplicación al respecto. El Ayuntamiento de Burgos (www.aytoburgos.es) no proporciona información en su página web sobre las aplicaciones que ha desarrollado sobre movilidad o sistemas inteligentes de transporte, aunque hemos podido llegar a ellas gracias a los buscadores de las propias páginas de venta de aplicaciones. La web sí informa sobre tres aplicaciones ganadoras del I Concurso de APPS para dispositivos móviles, organizado en 2014, donde encontramos MóvilBur, de contenido similar a la aplicación desarrollada por el Ayuntamiento: Muévete es Burgos.

En el caso de Muévete es Burgos se puede afirmar que es una aplicación fácil de manejar, dirigida a mayores de tres años, con "información sobre aparcamientos, paradas de taxis, estado del tráfico y gasolineras" (App Store, 2015) desarrollada por el Ayuntamiento de Burgos. Cuenta con más de 100 descargas y una evaluación de 3,4 sobre 5 (según la opinión de 10 usuarios) mientras que MóvilBur, que se actualizó por última vez en 2014 cuenta con más de 500 descargas y una puntuación de 4,8 sobre 5 (sobre 25 usuarios). La última versión desarrollada para el sistema operativo iOS es más reciente que la de Google. Otras aplicaciones desarrolladas por el Ayuntamiento de Burgos son Navidad Burgos, Servicios Sociales del Ayuntamiento, Semana Santa Burgos, Muévete por el Empleo, Festival Enclave de Calle, Festival Folclore Burgos y Fiestas Sampedros, desarrolladas tanto para Android como iOS.

En Galicia, el caso de La Coruña es el de una ciudad que encabeza el grupo de trabajo dedicado a la innovación social, donde se abarcan temáticas como la accesibilidad, cultura y deporte, participación ciudadana, salud y teleasistencia, seguridad y gestión de servicios públicos de emergencias, turismo y ocio, educación y gobierno abierto y open data. La página web del Concello da Coruña/Ayuntamiento de La Coruña (www.coruna.es) cuenta con un portal dedicado a la ciudad inteligente donde ofrece información sobre los proyectos desarrollados en la ciudad, especialmente en las áreas de interacción con el ciudadano, ocio y turismo, tráfico y transporte urbano, educación y visitas guiadas con realidad aumentada. La mayoría de los proyectos desarrollados tiene que ver con turismo y ocio. De esta ciudad se han analizado dos aplicaciones: Eventos Smart Coruña y Visitas Guiadas Coruña.

En el caso de Eventos Smart Coruña, esta ofrece "información sobre la celebración de eventos culturales y deportivos de ámbito público y privado" (Google Play, 2015). No dispone de clasificación por edades y ha sido descargada por más de 5.000 usuarios que la han evaluado muy positivamente (4,1 sobre 5 en el caso de los usuarios de Android y 5 sobre 5 entre los usuarios de Apple). Se trata de una aplicación que cuenta con un tutorial para facilitar su manejo, que ofrece planes por categorías y fecha, de manera muy sencilla para el usuario. Es útil tanto para vecinos como para visitantes. Además, el Ayuntamiento también ha desarrollado para Android la aplicación Visitas Guiadas Coruña. Desarrollada exclusivamente para este sistema operativo, la aplicación, sin clasificación por edad del público al que se dirige y descargada por más de 1.000 usuarios, ofrece información de callejero GPS y visitas guiadas. Cuenta con diez rutas configuradas y la posibilidad de incluir rutas personales. Permite el registro de usuario y utiliza realidad aumentada, información sobre puntos de interés, mapa y audioguía. Es una

3 http://www.redciudadesinteligentes.es/grupos-trabajo/ 
aplicación con carácter social, ya que permite comentar, valorar, compartir y seleccionar las alertas útiles para el usuario. Cuenta con una puntuación de 4,1 sobre 5 , basada en la opinión de 32 personas.

El Ayuntamiento de Valencia ha desarrollado App Valencia, recomendada para mayores de tres años. Disponible tanto para Android como iOS, sirve como portal de instalación de todas las aplicaciones desarrolladas por el Ajuntament de València/Ayuntamiento de Valencia. Es la más descargada de las aplicaciones analizadas (con más de 10.000 usuarios) e "incluye información de transportes, información de proximidad, tráfico, acceso a la sede electrónica del ayuntamiento para trámites, agenda de la ciudad, realidad aumentada y avisos del Ayuntamiento" (Google Play, 2015). El consistorio valenciano, líder del grupo de trabajo sobre temáticas de Gobierno, Economía y Negocios también ha desarrollado la aplicación Museos, Monumentos y más Cultura para Android y la aplicación Valencia Direct to You exclusiva para el sistema operativo iOS, orientadas al ocio y turismo. Para el análisis se seleccionó la aplicación App Valencia porque, aunque no está centrada únicamente en el e-commerce o plataformas de pago NFC, sí presta servicios inteligentes como permitir el pago de multas e impuestos a través de la misma.

\section{Conclusiones}

El escenario tecnológico dentro de las smart cities se debe fundamentar en el desarrollo de aplicaciones que analicen y generen datos útiles para la ciudadanía. Así, entre las conclusiones generales, es preciso destacar que en este sentido son necesarias políticas de fomento y acción que propicien, tanto desde los poderes públicos, por ejemplo los ayuntamientos, como desde iniciativas privadas, el acceso y comprensión de todo lo que rodea a las ciudades inteligentes. De nada sirven los nuevos desarrollos para unos ciudadanos que con dificultad entienden y manejan los lenguajes y aplicaciones digitales o por el contrario desconocen la existencia de dichas aplicaciones como deja traslucir el bajo número de descargas que han obtenido las apps analizadas. En otros casos, por desgracia, no todas las aplicaciones que se desarrollan son útiles o interesantes y en ocasiones no son más que una prolongación adaptada de los contenidos de la página web corporativa o institucional, lo que le resta valor añadido a la app y genera duplicidad innecesaria de la información.

En cuanto a las conclusiones específicas es preciso señalar que, el mayor interés por parte de las instituciones públicas y privadas estaría centrado en el desarrollo de aplicaciones sobre turismo. Sin embargo, parece existir poco interés por el desarrollo de aplicaciones sobre Salud y teleasistencia, Educación, e-commerce y plataformas de pago NFC, unos contenidos de gran importancia para el bienestar, el progreso y el desarrollo económico de los ciudadanos. Después del análisis de contenido se ha evidenciado, en algunos casos, una ausencia de interés y compromiso como es el caso de Valladolid que no ha desarrollado ninguna aplicación pese a ser cabeza de uno de los grupos de trabajo de la RECl; por el contrario, otras ciudades han visto y valorado los nuevos horizontes que las smart cities han abierto a los ciudadanos como es el caso de Burgos, la cual tiene mucha presencia incluso en proyectos en los que no tiene compromiso con la RECI. Por último, se puede observar que a pesar de los avances tecnológicos, el desarrollo de contenidos para las smart cities es todavía incipiente ya que, aunque cada vez hay más iniciativas en esta materia, aún queda mucho por hacer. 


\section{DISERTACIONES}

AVANCES

Anuario electrónico
ISSN: $1856-9536$

Doi: http://dx.doi.org/10.12804/revistas.urosario.edu.co/disertaciones/a.5106

Volumen 10, Número 2 / Julio-diciembre 2017

Versión PDF para imprimir desde

http://revistas.urosario.edu.co/index.php/disertaciones

\section{Referencias}

1. Arcila-Calderón, C. et al. (2016). Técnicas big data: Análisis de textos a gran escala para la investigación científica y periodística. El profesional de la información 25, (4), 623-631. http://dx.doi.org/10.3145/ epi.2016.jul.12

2. App Store [Tienda de aplicaciones de Apple]. Disponible en https://itunes.apple.com/es/genre/ios/ id36? $m t=8$ (Pp. 13, párrafo 2)

3. Ajuntament de València/Ayuntamiento de Valencia (2016): www.valencia.es

4. Ayuntamiento de Burgos (2016): www.aytoburgos.es

5. Ayuntamiento de Valladolid (2016): www.valladolid.es

6. BOE [Boletín Oficial del Estado] (2005). Instrumento de Ratificación del Convenio sobre el Acceso a la Información, la Participación del Público en la Toma de Decisiones y el Acceso a la Justicia en Materia de Medio Ambiente, firmado en Aarhus (Dinamarca), el 25 de junio de 1998. Página oficial del Ministerio de Presidencia. Sección Agencia Estatal Boletín Oficial del Estado. Disponible en http://www.boe.es/buscar/ doc.php?id=BOE-A2005-2528 (última consulta: 7 de noviembre de 2015). (Pp. 10, párrafo 2)

7. Bonete-Vizcaíno, F. (2015). Smart cities y patrimonio cultural. Una integración necesaria para el desarrollo. Revista Telos. Cuadernos de Comunicación e Innovación, (102), 1-7.

8. Cabezuelo-Lorenzo, F. et al. (2016). Análisis de la información y documentación científica española sobre el fenómeno de las smart cities, el hábitat de los nativos digitales. Cuadernos de Documentación Multimedia (CDM). doi: http://dx.doi.org/10.5209/rev_CDMU.2016.v27.n1.53000

9. Castells, M. (2008). Comunicación, poder y contrapoder en la sociedad red (I). Los medios y la política. Revista Telos. Cuadernos de Comunicación e Innovación, (74), 13-24.

10. Concello da Coruña/Ayuntamiento de La Coruña (2016): www.coruna.gal

11. Fundación Telefónica (2011). Smart cities: Un primer paso hacia el internet de las cosas. Madrid.

12. Fundación Telefónica (2015). La sociedad de la información en España 2014. Madrid.

13. Herranz-de-la-Casa, J. M. et al. (2015). Latest Trends and Initiatives in Corporate Social Responsibility: A Communicational Analysis of Successful Cases of Arts and Culture in Spain. Catalan Journal of Communication \& Cultural Studies 7, (2), 217-229.

14. IBM \& Institute For Business Value (2011). Ciudades más inteligentes para un desarrollo sostenible. Cómo optimizar los sistemas de la ciudad en una economía basada en el talento. Disponible en http://www. ibm.com/smarterplanet/global/files/es__es_es__cities__ciudades_inteligentes_para_desarrollo_sostenible_0622.pdf

15. González-Oñate, C. et al. (2015). Use, Consumption and Knowledge of New Technologies by Elderly People in France, United Kingdom and Spain. Comunicar Journal-Revista científica iberoamericana de Comunicación y Educación, (45), 19-28.

16. Google Play [Tienda de aplicaciones de Google] Disponible en https://play.google.com/store/apps?gl= ES\&utm_source=emea_Med\&utm_medium=hasem\&utm_content=Jun1115\&utm_campaign=Evergreen\&pcampaignid=MKT-DR-emea-es-all-Med-hasem-ap-Evergreen-Jun1115-1-SKWS.HASEM_kwid_43700007365263116\&gclid=CNGG4eCi3skCFQ0oGQodL6AN_g\&gclsrc=ds\#/now (p. 14, parr. 2) 
17. Enerlis, Ernst y Young (Eds). (2012). Libro Blanco de las smart cities. Ferrovial y Madrid Network: Madrid.

18. MECD [Página oficial del Ministerio de Educación Cultura y Deporte] (2014). Reto 6. Europa en un mundo cambiante: Sociedades inclusivas, innovadoras y reflexivas Horizonte 2020. Disponible en http://www. mecd.gob.es/cultura-mecd/dms/mecd/cultura-mecd/areas-cultura/cooperacion/mc/pcc/oficina-subprograma-cultura/jornadas/europcreati2014/abril/RCastrillo08042014.pdf

19. Pallot, M., Trousse, B., Senach, B., Scapin, D. (2010). Living Lab Research Landscape: From User Centred Design and User Experience Towards User Co-creation, Position Paper, First Living Labs Summer School Inria, París. Disponible en http://link.springer.com/chapter/10.1007\%2F978-3-642-20898-0_31\#

20. Prensky, M. (2001). Digital Natives, Digital Inmigrants. On the horizon. MCN, University Press 9 (5).

21. RECI [Red de Ciudades Inteligentes] (2015): http://www.redciudadesinteligentes.es/ (pp. 5, párrafo 1).

22. Schaffers H., Komninos N., Pallot M., Trousse B., Nilsson M., Oliveira A. (2011) Smart Cities and the Future Internet: Towards Cooperation Frameworks for Open Innovation, en Domínguez et al. (2011). Future Internet Assembly 2011: Achievements and Technological Promises. Springer-Verlag: Berlín, pp. 431-446.

23. Sánchez-Martínez, M. \& Ibar-Alonso, R. (2015). Convergence and Interaction in the New Media: Typologies of Prosumers Among University Students. Communication \& Society 28, (2), 87-99.

\section{Nota}

Este trabajo ha sido realizado en el marco del proyecto de investigación titulado "Smart cities: problemas de accesibilidad a los contenidos digitales en ciudadanos de edad avanzada" (FUSP-BS-PPC17/2014), financiado por la Fundación Universitaria San Pablo CEU y la Fundación Universia-Banco Santander y con el visto bueno de la Agencia Nacional de Evaluación y Prospectiva (ANEP), unidad dependiente de la Dirección General de Investigación Científica y Técnica, dentro de la Secretaría General de Ciencia, Tecnología e Innovación de la Secretaría de Estado de Investigación, Desarrollo e Innovación, del Ministerio de Economía y Competitividad del Gobierno de España. El puesto de investigadora principal (IP) del proyecto corre a cargo de la profesora María Sánchez Martínez que lidera un equipo de investigación de seis personas. 